\title{
Opening of a Respiratory Intermediate Care Unit in a General Hospital: Impact on Mortality and Other Outcomes
}

\author{
Marco Confalonieria Roberto Trevisan ${ }^{a}$ Maja Demsar ${ }^{a} \quad$ Luca Lattuada \\ Cinzia Longo $^{a}$ Rossella Cifaldi $^{a}$ Mitja Jevnikar ${ }^{a}$ Mario Santagiuliana ${ }^{a}$ \\ Lucia Pelusi $^{b}$ Riccardo Pistellid \\ ${ }^{a}$ Department of Pneumology and Respiratory Intermediate Care Unit, and b Medical Administration, University \\ Hospital of Cattinara, Trieste, ' Medical Administration, Local Health Authority No. 3 of Friuli Regional Health Service, \\ Gemona, and ${ }^{\mathrm{d}}$ Respiratory Medicine Unit, Columbus Hospital, Catholic University, Rome, Italy
}

\section{Key Words}

Respiratory intermediate care unit · Acute respiratory failure $\cdot$ Acute exacerbation of chronic obstructive pulmonary disease $\cdot$ Community-acquired pneumonia . Mortality

\begin{abstract}
Background: Respiratory intermediate care units (RICUs) are specialized areas aimed at optimizing the cost-benefit ratio of care. No data exist about the impact of opening a RICU on hospital outcomes. Objectives: We wondered if opening a RICU may improve the outcomes of patients with acute respiratory failure (ARF), acute exacerbation of chronic obstructive pulmonary disease (AECOPD), or community-acquired pneumonia (CAP). Methods: We analyzed the discharge abstracts of 2,372 admissions to the RICU and internal medicine units (IMUs) for ARF, AECOPD, and CAP. The IMUs at the Hospital of Trieste comprise emergency and internal wards. In order to investigate the determinants of outcomes, a matched case-control study was performed using clinical records. Results: The in-hospital mortality rate was lower in the RICU vs. IMUs (5.4 vs. $19.1 \%, p=0.0001$ ). Statistical differences did not change when comparing the RICU with
\end{abstract}

(c) 2015 S. Karger AG, Basel

0025-7931/15/0903-0235\$39.50/0 the emergency and internal wards. After adjusting for potential confounders, the risk of death for patients with CAP, AECOPD, or ARF was significantly higher in the IMUs than in the RICU (OR 6.90, 3.19, and 6.7, respectively, $\mathrm{p}<0.04)$. Both the frequency of transfer to the ICU ( 6 vs. $12 \%, p=0.0001$, OR 0.38 ) and the hospital stay (9.3 vs. 12.1 days, $p=0.0001$ ) were reduced in patients admitted to the RICU compared to those admitted to non-RICUs. Significant differences were found in care management concerning chest physiotherapy, mechanical ventilation, antibiotics, and corticosteroids. Conclusions: The opening of a RICU may be advantageous to reduce in-hospital mortality, the need for ICU admission, and the hospital stay of patients with AECOPD, CAP, and ARF. Better use of care resources contributed to better patient management in the RICU.

(c) 2015 S. Karger AG, Basel

\section{Introduction}

Respiratory diseases are the leading causes of mortality in general hospitals [1]. Among the respiratory causes of hospitalization, acute exacerbation of chronic obstructive pulmonary disease (AECOPD) and community-acquired

\section{KARGER 125}

E-Mail karger@karger.com www.karger.com/res
Marco Confalonieri, MD

Department of Pneumology and RICU

University Hospital of Cattinara, Strada di Fiume 447

IT-34149 Trieste (Italy)

E-Mail marco.confalonieri@aots.sanita.fvg.it 
pneumonia (CAP) with acute respiratory failure (ARF) are the two most frequent respiratory conditions that may necessitate mechanical ventilation, and both can be fatal [1].

Patients with AECOPD have a short-term mortality ranging from 3 to $30 \%$, depending on the severity of the acute episode and the presence of risk factors for death [2]. CAP accounts for a reported mortality of hospitalized adults ranging from 5 to $15 \%$ [3]. Severely ill patients with ARF, AECOPD, or CAP should be admitted to intensive care units (ICUs); however, because of the limited availability and high cost of ICU beds, these patients are often admitted to other units or departments. Where patients with ARF are admitted is an important issue that has, to date, been insufficiently explored. Whereas a number of recent studies and international guidelines have focused on medical and/or supportive treatment options aimed at improving hospital survival of patients with ARF due to AECOPD or CAP, less attention has been paid to the inhospital management of these patients outside the ICU. Some European surveys have found an association between high-quality specialized hospital care and better outcomes for patients with AECOPD $[4,5]$. Respiratory intermediate care units (RICUs) were introduced in Europe to improve the management and prognosis of patients with ARF, regardless of its etiology [6]. RICUs are specialized dedicated areas that aim to optimize the costbenefit ratio in the care of these patients, mainly through the provision of a lower level of nursing intensity compared with general ICUs. The opening of a new RICU at the University Hospital of Cattinara in Trieste (a general hospital that lacked a specialized unit for respiratory diseases until 2003) provided an opportunity to retrospectively compare the impact of RICU care, as opposed to care in internal medicine units (IMUs), on the survival of patients hospitalized with ARF, AECOPD, or CAP.

\section{Materials and Methods}

We performed a two-step study on patients admitted to the University Hospital of Cattinara during the initial time period after the opening of the new RICU. Firstly, we analyzed the discharge abstracts of consecutive admissions to the RICU and IMUs for ARF, AECOPD, and CAP, comparing outcomes between RICU and IMUs. Furthermore, we performed a matched case-control study based on complete medical records in a subset of patients of the same study population with ARF in order to evaluate differences in care management.

\section{Study Population}

We studied all the consecutive patients admitted during the period of January 1, 2004 until March 31, 2005 to non-ICUs, non- surgical units at the University Hospital of Cattinara, and discharged with a main diagnosis of ARF, AECOPD, or CAP. In patients presenting multiple acute respiratory episodes during a 90 -day period, we only included the first episode, assuming the subsequent admissions to be related to it. The study was approved by the Ethics Committee of the Azienda Ospedali Riuniti di Trieste.

\section{Hospital Settings}

In this study, we included patients discharged from the following hospital settings: the RICU, the internal medicine wards, and the emergency medicine unit. The RICU is a 15-bed unit with 6 fully monitored beds equipped for mechanical ventilation, a nurse-topatient ratio of 1:4, a pulmonologist continuously on duty, and respiratory physiotherapists as members of the care team. The four internal medicine wards each have up to 60 beds, none of them equipped with a full monitoring device or mechanical ventilator, and they have a nurse-to-patient ratio of 1:10. Furthermore, at night, there is only one physician specialized in internal medicine on call for all four wards. The emergency medicine unit is not a short-stay unit. It has 24 beds, 12 of which are fully monitored and equipped for mechanical ventilation, a nurse-to-patient ratio of 1:4, and a physician specialized in emergency and internal medicine continuously on duty; however, there are no respiratory physiotherapists in the care team. All patients included in this study were initially assessed in the hospital's emergency room and promptly admitted to the different settings according to the availability of beds. Patients with end-stage disease (e.g. stroke with severe functional limitations, metastatic cancer, etc.) and/or palliative treatment (e.g. DNR or DNI) were excluded as they were prone to selection bias.

\section{Data Source}

We first evaluated the hospital discharge abstracts of all patients with ARF, AECOPD, or CAP. Discharge abstracts are routinely collected by the hospital information system of the Regional Health Service of the Friuli-Venezia Giulia Region, Italy, where Trieste is located. They contain patient demographics, admission and discharge dates, up to six discharge diagnoses (International Classification of Disease, rev 9, clinical modification, ICD9-CM), up to six clinical procedures, and status at discharge (alive, deceased, or transferred to another hospital). Medical records containing clinical information and medical interventions are available in the hospital data set.

\section{Outcomes}

The primary outcome was in-hospital death for each acute respiratory episode during the study period for patients with a main diagnosis of AECOPD, CAP, or ARF. Furthermore, transfers to ICUs were identified from hospital discharge abstracts. The length of in-hospital stay for each episode was also measured. Potential confounders for mortality, such as age, length of stay, and major comorbidities (chronic heart disease, diabetes, chronic renal disease, chronic liver disease, chronic respiratory disease other than COPD, hypertension, psychiatric disease, cancer, neuromuscular disorders, and obesity) were considered in order to calculate the between-unit adjusted odds ratios (ORs).

Case-Control Study in Patients Admitted to Different Hospital Settings

In order to investigate the potential determinants of different outcomes, we evaluated the between-unit differences in care man- 
Table 1. Characteristics and outcomes of patients with ARF, AECOPD, or CAP according to hospital units

\begin{tabular}{|c|c|c|c|c|c|c|c|c|}
\hline & $\begin{array}{l}\text { Study } \\
\text { population }\end{array}$ & RICU & IMUs & $\begin{array}{l}\mathrm{p} \\
\text { value }\end{array}$ & $\begin{array}{l}\text { Emergency } \\
\text { unit }\end{array}$ & $\begin{array}{l}\mathrm{p} \\
\text { value }\end{array}$ & $\begin{array}{l}\text { Internal } \\
\text { medicine wards }\end{array}$ & $\begin{array}{l}\mathrm{p} \\
\text { value }\end{array}$ \\
\hline Patients & 2,372 & 647 & 1,725 & & 447 & & 1,278 & \\
\hline Males & 1,312 & 368 & 944 & & 245 & & 699 & \\
\hline Females & 1,060 & 279 & 781 & & 202 & & 579 & \\
\hline In-hospital mortality & $365(15.3)$ & $35(5.4)$ & $330(19.1)$ & 0.0001 & $64(14.3)$ & 0.0001 & $266(20.8)$ & 0.0001 \\
\hline Need for ICU admission & $254(10.7)$ & $39(6.02)$ & $215(12.4)$ & 0.0001 & $58(12.9)$ & 0.0001 & $157(12.2)$ & 0.0001 \\
\hline Previous chronic heart disease & $897(37.8)$ & $226(34.9)$ & $671(38.8)$ & 0.090 & $168(37.5)$ & 0.414 & $503(39.3)$ & 0.067 \\
\hline Acute heart disorders & $609(25.6)$ & $154(23.8)$ & $455(26.3)$ & 0.234 & $113(25.2)$ & 0.647 & $342(26.7)$ & 0.187 \\
\hline Diabetes & $319(13.4)$ & 77 (11.9) & $242(14.0)$ & 0.205 & $51(11.4)$ & 0.875 & $191(14.9)$ & 0.084 \\
\hline Chronic renal disease & $99(4.1)$ & $19(2.9)$ & $80(4.6)$ & 0.083 & $19(4.2)$ & 0.321 & $61(4.7)$ & 0.078 \\
\hline Acute renal disease & $47(1.9)$ & $11(1.7)$ & $36(2.0)$ & 0.759 & $14(3.1)$ & 0.186 & $22(1.7)$ & 0.852 \\
\hline Chronic liver disease & $59(2.4)$ & $11(1.7)$ & $48(2.7)$ & 0.208 & $14(3.1)$ & 0.186 & $34(2.8)$ & 0.186 \\
\hline Neuromuscular disorders & $147(8.5)$ & $59(9.1)$ & $88(5.0)$ & 0.0001 & $41(9.1)$ & 0.915 & $47(3.6)$ & 0.0001 \\
\hline Obesity & $205(8.6)$ & $69(10.6)$ & $132(7.6)$ & 0.024 & $48(10.7)$ & 0.963 & $84(6.5)$ & 0.002 \\
\hline
\end{tabular}

Data from hospital discharge abstracts are presented as $\mathrm{n}(\%)$ or mean \pm SD. The p values are related to the difference between RICU and each non-RICU data. LOS surv = Length of hospital stay for patients who survived; LOS all = length of hospital stay for all patients.

agement in a case-control study conducted on a subsample of acute respiratory episodes selected from the overall data set. First, all episodes of in-hospital death in the RICU and matched control episodes of in-hospital death in IMUs (the emergency medicine unit and internal medicine wards) were included. The matching criteria for control selection were age (within a range of 4 years), APACHE II score [7] within 3, $\mathrm{PaO}_{2}$ within $5 \mathrm{~mm} \mathrm{Hg}, \mathrm{PaCO}_{2}$ within $8 \mathrm{~mm} \mathrm{Hg}$, pH within $0.03, \mathrm{PaO}_{2} / \mathrm{FiO}_{2}$ within 20 , Charlson comorbidity index [8] within 0.6 , and lung injury score [9] within 0.5 of that of a patient admitted to the RICU with the same main diagnosis. At least seven of the eight criteria used for matching had to be fulfilled to select a control case.

The final sample size was calculated as the sample size needed to obtain between-unit significant differences in the mortality rate according to the mean value and variance of that difference in our data set. The calculated sample size was reached by adding to both cases and controls matched episodes with a nonfatal evolution in the RICU and IMUs. For each patient, we analyzed the following parameters: demographics, blood gases, $\mathrm{PaO}_{2} / \mathrm{FiO}_{2}$ ratio, APACHE II score [7] at admission and the Charlson index to assess the weight of comorbidities [8], lung injury score [9], time to the first and second blood gas analysis, time to initiation of antibiotics, use of noninvasive ventilation (NIV) and/or chest physiotherapy, and administration of corticosteroids.

\section{Statistical Analysis}

Continuous variables were expressed as the mean \pm standard deviation or as the median (interquartile range) according to data distribution. Categorical variables were presented as numbers and percentages. Between-unit differences in patient characteristics and care management were evaluated with analysis of variance or the Mann-Whitney $U$ test for continuous data and with the $\chi^{2}$ test for categorical variables. Logistic regression was used to evaluate the independent effect of the hospital setting on mortality and ICU transfers, adjusting for age, length of stay, and major comorbidities. The StatView package was used for data management and analysis (version 5.01; SAS Institute Inc., Cary, N.C., USA). All tests were two-tailed, and a $\mathrm{p}$ value of $<0.05$ was considered significant.

\section{Results}

\section{Observational Cohort Study on Mortality Based on \\ Data from Hospital Discharge Abstracts}

Overall, we identified 2,372 acute respiratory patients in the period of January 1, 2004 to March 31, 2005 among patients admitted to the University Hospital of Trieste with the main diagnoses of AECOPD, ARF, or CAP. Table 1 shows the patients' characteristics, diagnoses, and in-hospital outcomes according to the different hospital settings. CAP was the first diagnosis in 1,087 patients, of whom 117 were admitted to the RICU and 970 to IMUs. ARF was the first diagnosis in 685 patients, with 463 of them admitted to the RICU and 222 to IMUs. AECOPD was the first diagnosis in 600 patients, with 67 admitted to the RICU and 533 to IMUs. Major comorbidities and gender were not significantly different between the pa- 
Table 2. In-hospital death and transfer to ICU by patients' main diagnosis (CAP, AECOPD, or ARF) and hospital unit

\begin{tabular}{llcc}
\hline & Patients & In-hospital death & Transfer to ICU \\
\hline CAP & & & \\
RICU & $117(18.0)$ & $5(4.3)^{*}$ & $7(6.0)^{*}$ \\
Emergency unit & $187(41.8)$ & $36(19.2)$ & $28(14.9)$ \\
Internal medicine wards & $783(61.2)$ & $195(24.9)$ & $76(9.7)$ \\
IMUs & 970 & $231(23.8)$ & $104(10.7)$ \\
\hline AECOPD & & & $5(7.5)^{*}$ \\
RICU & $67(10.3)$ & $2(3.0)^{*}$ & $16(13.3)$ \\
Emergency unit & $120(30.8)$ & $11(9.1)$ & $56(13.5)$ \\
Internal medicine wards & $413(32.3)$ & $48(8.9)$ & $72(13.5)$ \\
IMUs & 533 & $48(9.0)$ & $27(5.8)^{*}$ \\
\hline ARF & & $28(6.0)^{*}$ & $17(15.7)$ \\
RICU & $463(71.5)$ & $17(15.7)$ & $22(19.2)$ \\
Emergency unit & $108(24.1)$ & $32(28.0)$ & $39(17.6)$ \\
Internal medicine wards & $114(8.9)$ & $49(22.1)$ & \\
IMUs & 222 & & \\
\hline
\end{tabular}

Data are presented as $\mathrm{n}$ (\% of the total number in the same line).

* Significantly lower frequency with reference to both non-RICU unit types $(\mathrm{p}<0.05)$.

Table 3. RICU versus non-RICU ORs for in-hospital mortality adjusted for age, gender, length of stay, and comorbidities in the IMUs and the RICU

\begin{tabular}{lll}
\hline Main diagnosis & Adjusted OR $(95 \% \mathrm{CI})$ & p value \\
\hline CAP & $5.78(1.97-13.16)$ & 0.001 \\
AECOPD & $2.65(0.614-11.34)$ & 0.037 \\
ARF & $5.67(3.23-10.24)$ & 0.001 \\
\hline
\end{tabular}

tients admitted to the RICU and those admitted to IMUs. However, patients admitted to IMUs were slightly but significantly older, whereas patients admitted to the RICU and the emergency unit had a higher frequency of chronic respiratory diseases other than COPD, obesity, and neuromuscular disorders. Chronic respiratory diseases other than COPD were more often combined in the patient population admitted to the RICU (table 1).

The in-hospital mortality rate and the frequency of transfer to the general ICU for intubation were significantly lower in the patients admitted to the RICU in comparison with both the emergency unit and the internal medicine wards (table 1). In both the overall and survivor groups, the hospital stay was significantly shorter in patients admitted to the RICU than in those treated in
IMUs, with no differences between the emergency unit and the internal medicine wards (table 1).

The significantly lower frequencies of death and transfer to the general ICU in patients admitted to the RICU were confirmed in subgroups of patients classified according to their main diagnosis (table 2).

The adjusted ORs for in-hospital mortality are reported in table 3, comparing the patients admitted to the RICU and those admitted to IMUs. After adjusting for potential confounders (age, gender, and comorbidities), there was a clear and statistically significant increased risk of death for the patients admitted to IMUs, regardless of whether they were affected by CAP, AECOPD, or ARF (table 3). Moreover, the adjusted risk of transfer to the general ICU was lower in the patients admitted to the RICU than in those admitted to IMUs (OR 0.38, 95\% CI $0.27-0.59$ ).

\section{Control-Matched Study in a Sample of Patients according to Hospital Setting}

In the case-control study, a sample of 180 patients selected from the already described cohort of 2,372 patients was studied. In this cohort, 1,274 patients had complete clinical records and met all the matching criteria.

Only 31 of the 35 patients who died in the RICU had complete records and met all the matching criteria. Of the 
Table 4. Characteristics of patients included in the case-control study according to hospital setting

\begin{tabular}{|c|c|c|c|c|}
\hline & RICU & $\begin{array}{l}\text { Emergency } \\
\text { unit }\end{array}$ & $\begin{array}{l}\text { Internal } \\
\text { medicine wards }\end{array}$ & $\begin{array}{l}\mathrm{p} \\
\text { value }\end{array}$ \\
\hline Patients included, $\mathrm{n}$ & 60 & 58 & 62 & \\
\hline Dead patients with ARF, AECOPD, or CAP, $n$ & 31 & 29 & 33 & \\
\hline Age, years & $69.8(8.1)$ & $73.1(7.2)$ & $71.9(8.4)$ & 0.075 \\
\hline Male/female ratio & 1.81 & 1.63 & 2.2 & \\
\hline Charlson comorbidity index & $7.7(3.8)$ & $7.8(4.1)$ & $7.6(3.9)$ & 0.965 \\
\hline $\mathrm{PaO}_{2}$ at admission, $\mathrm{mm} \mathrm{Hg}$ & $59(6.0)$ & $58.7(8.4)$ & $60.1(10.3)$ & 0.631 \\
\hline $\mathrm{PaCO}_{2}$ at admission, $\mathrm{mm} \mathrm{Hg}$ & $61.3(29.7)$ & $54.1(18.4)$ & $52.7(12.7)$ & 0.063 \\
\hline $\mathrm{pH}$ at admission & $7.33(0.14)$ & $7.34(0.09)$ & $7.34(0.09)$ & 0.846 \\
\hline $\mathrm{PaO}_{2} / \mathrm{FiO}_{2}$ at admission, $\mathrm{mm} \mathrm{Hg}$ & $199(67.8)$ & $201(76.9)$ & $223(48.8)$ & 0.082 \\
\hline Lung injury score & $2.5(0.9)$ & $2.4(1.2)$ & $2.2(1.4)$ & 0.097 \\
\hline APACHE II score & $22.5(5.9)$ & $21.4(7.1)$ & $19.8(6.4)$ & 0.071 \\
\hline DRG weight & $1.69(0.6)$ & $1.61(0.3)$ & $1.35(0.5)$ & $0.001^{*}$ \\
\hline
\end{tabular}

All data derived from medical records are expressed as means (standard deviation), or as medians (interquartile range) according to data distribution.

* The DRG weight of patients admitted to the RICU and emergency unit was significantly higher than that of patients admitted to the internal medicine wards.

Table 5. Management attitude and treatment timing in a sample of matched patients with ARF admitted in different hospital setting

\begin{tabular}{lcccr}
\hline & RICU & $\begin{array}{l}\text { Emergency } \\
\text { unit }\end{array}$ & $\begin{array}{l}\text { Internal } \\
\text { medicine wards }\end{array}$ & $\mathrm{p}$ \\
\hline Median time to second blood gas check, h & $1.56(0.4)$ & $4.26(3.4)$ & $17.1(10.9)$ & $<0.0001$ \\
Mean time to antibiotics initiation, h & $0.84(0.3)$ & $1.63(1.6)$ & $2.2(2.12)$ & $<0.0001$ \\
Median time to mechanical ventilation, days & $0.3(0.6)$ & $0.7(0.7)$ & $4.8(3.2)$ & 0.0001 \\
\hline Use of NIV, n (\%) & $42(6.02)$ & $27(12.45)$ & $12(12.26)$ & 0.0001 \\
Use of corticosteroids, n (\%) & $58(96.6)$ & $46(79.3)$ & $39(62.9)$ & 0.0012 \\
Use of chest physiotherapy, n (\%) & $43(71.6)$ & $11(18.9)$ & $6(9.6)$ & 0.0001 \\
\hline
\end{tabular}

Data in parentheses are interquartile ranges or standard deviations unless otherwise indicated.

330 patients who died in IMUs, 62 patients fulfilling all the matching criteria were selected ( 29 from the emergency medicine unit and 33 from other internal medicine wards). A sample of 87 survivors ( 29 from each unit) was selected using the same matching criteria and included in the case-control study in order to reach the planned sample size. Patient characteristics in the three different hospital settings are reported and compared in table 4. Due to the use of matching criteria, there were no statistically significant differences in age, comorbidity index, arterial $\mathrm{pH}$ and $\mathrm{PaCO}_{2}$ at admission, $\mathrm{PaO}_{2} / \mathrm{FiO}_{2}$ ratio, and APACHE II score between the different groups. The average diagnosis-related group (DRG) weight in the
RICU, emergency medicine unit, and internal medicine wards was $198.2 \pm 191.3,215 \pm 194.9$, and $88.3 \pm 3.9$, respectively. The difference in DRG was not significant between the RICU and the emergency unit, whilst it was significant between the internal medicine wards and both the RICU and the emergency unit $(\mathrm{p}=0.001)$.

The use of corticosteroids, NIV, antibiotics, physiotherapy, and blood gas tests in the RICU and the other hospital settings is compared in table 5 .

The time to the second blood gas test, the interval before starting antibiotic therapy, and the interval before initiating mechanical ventilation were shorter in the RICU than in the IMUs (table 5). Moreover, physiother- 
apy was used in most patients admitted to the RICU but only in a minority of those admitted to the IMUs. Corticosteroids for AECOPD, CAP, and ARF were also used more frequently in the RICU than in the other settings.

\section{Discussion}

The main finding of our study is that the presence of RICUs in general teaching hospitals may be advantageous in terms of reductions in in-hospital mortality rates, the need for ICU admission, and the length of hospital stay for patients with a main diagnosis of AECOPD, CAP, and ARF. Patients managed at the RICU had a significantly reduced mortality in comparison with both the emergency unit and the internal medicine wards. Our study also suggests that RICU admission may be associated with shorter times to the application of NIV and the administration of specific drugs, and more frequent arterial gas monitoring and chest physiotherapy, in comparison with what occurs in IMUs in the same hospital. From an organizational perspective, the RICU and the emergency unit may seem to differ much less as compared with internal medicine wards, but the RICU showed significantly different outcomes with regard to both emergency and internal settings. A dedicated specialized approach to acute respiratory diseases can make the difference. Our finding of better survival in patients with AECOPD and CAP admitted to a RICU is in keeping with the results of other studies from Europe and North America, which suggest that a specialized organization of care and the availability of resources are key factors in obtaining improved outcomes $[4,5,10-12]$.

Most of the studies comparing survival in patients with AECOPD and CAP applied benchmark methods to establish the quality of care in different hospitals. On the other hand, very few evaluated the impact of the different organization of care between different units within the same hospital after the opening of a new specialized unit devoted to respiratory patients. Franklin et al. [13] reported that mortality decreased on medical wards after the opening of a RICU, but no data were provided about mortality during the first months of operation of the new RICU. Fox et al. [14] demonstrated that opening a RICU helped to reduce readmissions to a general ICU.

The experience of our hospital shows that opening a RICU may increase the survival rate of patients admitted with ARF, AECOPD, and CAP. Furthermore, the RICU organizational model may improve the management of patients with ARF due to common causes, reducing the length of stay and the need for ICU admission and proving less costly than the ICU model, yet without affecting the outcome $[15,16]$.

To date, NIV remains established as the first choice for mechanical ventilation of patients with AECOPD, and it is widely recommended that it be delivered in dedicated units by operators trained and experienced in its application [17]. The main advantage of the RICU for patients with AECOPD may be the timely and accurate use of NIV. According to the European Respiratory Society, the RICU (thanks to the presence of NIV-dedicated staff, the monitoring provided, and the expertise available) is an example of good management of hospital resources and allows the best outcome for AECOPD patients [6]. Nevertheless, some patients with AECOPD are still admitted to hospital units where NIV is not routinely available and used [18-20]. Our study confirms that the prompt and safe use of NIV may constitute an advantage of RICU admission for patients with AECOPD, added to the expertise and the better facilities that RICUs offer these patients. A British national audit on AECOPD showed that units run by respiratory specialists with a standardized high quality of care indeed record lower mortality rates and shorter hospital stays [21]. Since 1999, the Royal College of Physicians has recommended that at least four respiratory specialists per 1,000 hospital beds should be available in order to reduce AECOPD mortality rates [22]. Moreover, in hospitals that do not have a RICU, some patients are admitted to ICUs at higher costs and without any improvement of outcomes [23]. In our study, we demonstrated that RICU admission led to improved survival in CAP and ARF patients, too. There are two possible specific explanations for the better outcome of CAP in patients admitted to the RICU: the time to the initiation of antibiotics was shorter and the use of corticosteroids was higher in the RICU than in the non-RICU settings. Timely and effective antimicrobial therapy is crucial in optimizing the outcomes of patients with CAP [24]. Moreover, the ready availability of fiberoptic bronchoscopy in RICUs may facilitate the recognition of the pathogens responsible for the most severe cases of CAP in order to guide the antibiotic therapy [25].

The use of corticosteroids in CAP remains controversial in the literature [26]; however, some years after a previous randomized controlled trial [27], which showed a reduction of hospital mortality among severe CAP patients treated with prolonged low doses of corticosteroids compared with conventional therapy and placebo, our personal experience continues to be positive. Our study also showed that the patients admitted to the RICU 
regularly received chest physiotherapy, whilst those admitted to IMUs did so only occasionally. The use of NIV was found to be similar in the emergency unit and the RICU, but patients admitted to the RICU underwent blood gas checks earlier than those treated in the other settings.

In Italy, the increasing prevalence of acutely decompensated respiratory diseases in the face of a shortage of ICU beds has prompted a growing interest in RICUs [28]. The last three decades have seen an increase in both the number and the expertise of Italian RICUs, with a shift toward less expensive care and greater complexity of interventions and patient dysfunctions [29]. A recent national survey found that the patients most frequently admitted to Italian RICUs were those with acute-onchronic respiratory failure, particularly AECOPD, obesity hypoventilation, and chest-wall disease [29]. The present study, on the other hand, showed a higher frequency of RICU admissions for ARF and CAP, revealing that the Trieste hospital pulmonologists choose to manage a certain category of critically ill patients who, in other Italian hospitals, are usually managed by intensivists or emergency physicians [30].

There currently exist various hospital care models for patients with ARF. The present study showed that opening a RICU in a general hospital may be an advantageous move also for patients with CAP and ARF. Before the opening of the RICU at the teaching hospital in Trieste, the pulmonology ward was located in a separate building - an old sanatorium previously devoted mainly to the treatment of patients affected by tuberculosis. The results of our study suggest that opening a RICU in a general hospital is useful for obtaining superior outcomes in acute patients. Furthermore, the new unit required practically no investment from the hospital, given that the savings made by closing the old sanatorium largely covered the costs of opening the RICU.

\section{Study Limitations}

The limitations of this study are related to its observational design, the time elapsing between the occurrence of the acute respiratory episodes and the data analysis, and the lack of 30-day mortality data. Generally speaking, prospective pragmatic clinical trials of health service interventions may also present bias and limitations as already observed, e.g., in the field of stroke management [31]. For example, assessments in prospective trials cannot be truly blinded, because location identifies allocation and observers might rate the care provided in one unit more favorably than that provided in others. In the pres- ent context, the assessment could have been influenced by patient allocation to emergency department care and/ or by the physicians' decision to transfer patients to the ICU or to the RICU. Just after the opening of a new RICU, the emergency room team referred the most severe respiratory patients to the RICU or emergency unit on a bed availability basis only, whereas the less severe patients were admitted to medical wards. In fact, the differences in the DRG figures were not significant between the RICU and the emergency unit, but only internal medicine wards reported a reduced DRG weight compared with the other units.

The main advantage of the present study's design is that it gives insight into a real-life situation with organizational implications for the management of acute respiratory patients in general hospitals. It must also be pointed out that treatments for patients with AECOPD [32], CAP [33], and ARF have not substantially changed over the past 10 years, with the exception of the introduction of extracorporeal membrane oxygenation, which is reserved for a limited number of ARF cases refractory to the usual therapy, most of them directly admitted to general ICUs. Thirty-day mortality could have better face validity than in-hospital mortality. In fact, a premature hospital discharge could limit the in-hospital mortality assessment, while a prolonged hospitalization and related injuries may affect in-hospital mortality data [34]. Nevertheless, the measure selected for internal benchmarking should depend primarily on the data linkage capabilities of the health care system and less on mortality timing [35].

However, even taking into account the above limitations, the reduction of the mortality rate in the RICUtreated patients was quite large and is an interesting finding that merits further attention.

\section{Conclusions}

The opening of a RICU in a general hospital may be advantageous not only to improve the in-hospital mortality, but also to decrease the frequency of transfers to general ICUs and the hospital stay of patients with AECOPD, $\mathrm{CAP}$, and ARF. Better use of mechanical ventilation, antibiotic treatment, corticosteroids, and care resources seems to be the key factor contributing to successful patient management in this setting. 


\section{References}

1 Hall MJ, Levant S, DeFrances CJ: Trends in Inpatient Hospital Deaths: National Hospital Discharge Survey, 2000-2010. NCHS data brief, No. 118. Hyattsville National Center for Health Statistics, 2013.

-2 Singanayagam A, Schembri S, Chalmers JD: Predictors of mortality in hospitalized adults with acute exacerbation of chronic obstructive pulmonary disease. Ann Am Thorac Soc 2013;10:81-89.

3 Aliberti S, Brambilla AM, Chalmers JD, et al: Phenotyping community-acquired pneumonia according to the presence of acute respiratory failure and severe sepsis. Respir Res 2014; 4:15-27.

4 Roberts CM, Barnes S, Lowe D, et al: Evidence for a link between mortality in acute COPD and hospital type and resources. Thorax 2003; 58:947-949.

5 Agabiti N, Belleudi V, Davoli M, et al: Profiling hospital performance to monitor the quality of care: the case of COPD. Eur Respir J 2010;35:1031-1038.

$\checkmark 6$ Corrado A, Roussos C, Ambrosino N, et al: Respiratory intermediate care units: a European survey. Eur Respir J 2002;20:1343-1350.

$>7$ Knaus WA, Draper EA, Wagner DP, Zimmermann JE: APACHE II: a severity of disease classification system. Crit Care Med 1985; 13:818-829.

$>8$ Charlson ME, Pompei P, Ales KL, McKenzie CR: A new method of classifying prognostic comorbidity in longitudinal studies: development and validation. J Chronic Dis 1987;40: 373-383.

$\checkmark 9$ Murray JF, Matthay MA, Luce JM, et al: An expanded definition of the adult respiratory distress syndrome. Am Rev Respir Dis 1988; 138:720-723.

10 Connolly MJ, Lowe D, Anstey K, et al: Admissions to hospital with exacerbations of chronic obstructive pulmonary disease: effect of age related factors and service organisation. Tho$\operatorname{rax} 2006 ; 61: 843-848$

-11 Polanzyk CA, Lane A, Coburn M, et al: Hospital outcomes in major teaching, minor teaching and non-teaching hospitals in New York State. Am J Med 2002;112:255-261.

12 Slenter RHJ, Sprooten RTM, Wesseling G, et al: Predictors of 1-year mortality at hospital admission for acute exacerbations of chronic obstructive pulmonary disease. Respiration 2013;85:15-26.
13 Franklin CM, Rackow EC, Mamdani B, Nightingale S, Burke G, Weil MH: Decreases in mortality on a large urban medical service by facilitating access to critical care. An alternative to rationing. Arch Intern Med 1988; 148:1403-1405.

14 Fox AJ, Owen-Smith O, Spiers P: The immediate impact of opening an adult high dependency unit on intensive care unit occupancy. Anaesthesia 1999;54:280-283.

15 Bertolini G, Confalonieri M, Rossi C, et al: Costs of the COPD. Differences between the intensive care unit and respiratory intermediate care unit. Respir Med 2005;99:894-900.

16 Elpern EH, Silver MR, Rosen RL, Bone RC: The noninvasive respiratory care unit. Patterns of use and financial implications. Chest 1991;99:205-208.

17 Global Initiative for Chronic Obstructive Lung Disease: Global strategy for the diagnosis, management, and prevention of chronic obstructive pulmonary disease; updated 2014. www.goldcopd.org/guidelines-globalstrategy-for-diagnosis-management.html (accessed February 20, 2014).

18 Plant PK, Owen JL, Elliott MW: One year period prevalence study of respiratory acidosis in acute exacerbations of COPD: implications for the provision of non-invasive ventilation and oxygen administration. Thorax 2000;55: 550-554.

19 Maheshwari V, Paioli D, Torhaar R, Hill NS: Utilization of noninvasive ventilation in acute care hospitals: a regional survey. Chest 2006; 129:1226-1233.

20 Burns K, Sinuff T, Adhikari N, et al: Bilevel noninvasive positive pressure ventilation for acute respiratory failure: survey of Ontario practice. Crit Care Med 2005;33:1477-1483.

21 Price LC, Lowe D, Hosker HSR, et al: UK National COPD Audit 2003: impact of hospital resources and organization of care on patient outcome following admission for acute COPD exacerbation. Thorax 2006;61:837842.

22 Royal College of Physicians: Working for $\mathrm{Pa}$ tients, Working Party Document. London, Royal College of Physicians, 1999.

23 Byrick RJ, Mazer D, Caskennette GM: Closure of an intermediate care unit. Impact on critical care utilization. Chest 1993:104:876881 .
24 Houck PM, Bratzler DW, Niederman M, et al: Pneumonia treatment process and quality. Arch Intern Med 2002;162:843-844.

25 Mandell LA, Wunderink RG, Anzueto A, et al: Infectious Disease Society of America/ American Thoracic Society consensus guidelines on the management of community-acquired pneumonia in adults. Clin Infect Dis 2007;44:S27-S72.

26 Confalonieri M, Meduri GU: Glucocorticoid treatment in community-acquired pneumonia. Lancet 2011;377:1982-1984.

27 Confalonieri M, Urbino R, Potena A, et al: Hydrocortisone infusion for severe community-acquired pneumonia: a preliminary randomized study. Am J Respir Crit Care Med 2005; 171:242-248.

$\checkmark 28$ Confalonieri M, Gorini M, Ambrosino N, et al: Respiratory intensive care units in Italy: a national census and prospective cohort study. Thorax 2001;56:373-378.

29 Scala R, Corrado A, Confalonieri M, et al: Increased number and expertise of Italian respiratory high-dependency care units: the second national survey. Respir Care 2011;56: 1100-1107.

30 Crimi C, Noto A, Princi P, et al: Survey of non-invasive ventilation practices: a snapshot of Italian practice. Minerva Anestesiol 2011; 77:971-978.

-31 Evans A, Perez I, Harraf F, et al: Can differences in management processes explain different outcomes between stroke unit and stroke team care? Lancet 2001;358:15861592.

32 Confalonieri M, Aiolfi S, Gandola L, et al: Severe exacerbations of chronic obstructive pulmonary disease treated with $\mathrm{BiPAP}^{\circledR}$ by nasal mask. Respiration 1994;61:310-316.

33 Kohno S, Seki M, Takehara K, et al: Prediction of requirement for mechanical ventilation in community-acquired pneumonia with acute respiratory failure: a multicenter prospective study. Respiration 2013;85:27-35.

34 Zhan C, Miller MN: Excess length of stay, charges, and mortality attributable to medical injuries during hospitalization. JAMA 2003; 290:1868-1874.

35 Borzecki AM, Christiansen CL, Chew P, et al: Comparison of in-hospital versus 30-day mortality assessments for selected medical conditions. Med Care 2010;48:117-121. 


\section{Erratum}

Table 5 in the paper by Confalonieri $\mathrm{M}$ et al. entitled 'Opening of a respiratory intermediate care unit in a general hospital: impact on mortality and other outcomes' [Respiration 2015;90:235-242] should read:

Table 5. Management attitude and treatment timing in a sample of matched patients with ARF admitted in different hospital setting

\begin{tabular}{lccrr}
\hline & RICU & $\begin{array}{l}\text { Emergency } \\
\text { unit }\end{array}$ & $\begin{array}{l}\text { Internal } \\
\text { medicine wards }\end{array}$ & $\mathrm{p}$ \\
\hline Median time to second blood gas check, h & $1.56(0.4)$ & $4.26(3.4)$ & $17.1(10.9)$ & $<0.0001$ \\
Mean time to antibiotics initiation, h & $0.84(0.3)$ & $1.63(1.6)$ & $2.2(2.12)$ & $<0.0001$ \\
Median time to mechanical ventilation, days & $0.3(0.6)$ & $0.7(0.7)$ & $4.8(3.2)$ & 0.0001 \\
\hline Use of NIV, n (\%) & $42(70.0)$ & $27(46.5)$ & $12(19.3)$ & 0.0001 \\
Use of corticosteroids, n (\%) & $58(96.6)$ & $46(79.3)$ & $39(62.9)$ & 0.0012 \\
Use of chest physiotherapy, n (\%) & $43(71.6)$ & $11(18.9)$ & $6(9.6)$ & 0.0001 \\
\hline
\end{tabular}

Data in parentheses are interquartile ranges or standard deviations unless otherwise indicated. 